\title{
Gut microbiota and metabolic disorders: how prebiotic can work?
}

\author{
Nathalie M. Delzenne*, Audrey M. Neyrinck and Patrice D. Cani \\ Université catholique de Louvain, Louvain Drug Research Institute, Metabolism and Nutrition Research Group, Avenue \\ Mounier 73, Box B1.73.11, B-1200 Brussels, Belgium
}

\begin{abstract}
Experimental data in animals, but also observational studies in obese patients, suggest that the composition of the gut microbiota differs in obese $v$. lean individuals, in diabetic $v$. non-diabetic patients or in patients presenting other diseases associated with obesity or nutritional dysbalance, such as non-alcoholic steatohepatitis. In the present review, we will describe how changes in the gut microbiota composition and/or activity by dietary fibres with prebiotic properties, can modulate host gene expression and metabolism. We will evaluate their potential relevance in the management of obesity and related metabolic disturbances, in view of the experimental data and intervention studies published up to date.
\end{abstract}

Key words: Gut microbiota: Obesity: Prebiotics: Inflammation: Gut peptides

The worldwide epidemic of obesity is a crucial problem of public health, as it is associated with a cluster of metabolic disorders such as insulin resistance, type 2 diabetes and fatty liver disease $^{(1)}$. The cause of obesity is basically linked to 'nutritional disequilibrium' in an individual who consumes an excess of fat and calories $v$. energy expenditure, over a relatively long period of time. In addition, overfeeding is often associated with inadequate nutrition, leading namely to a low intake of n-3 PUFA and of dietary fibres. In that respect, some dietary habits related to an increase in bioactive food components present in whole grain cereals could be helpful in prevention of chronic diseases ${ }^{(2)}$. In recent years, there has been increased attention focused on the bacteria that colonise our gut, which in ideal conditions live in symbiosis with the host ${ }^{(3,4)}$. Novel culture-independent technologies based on the analysis of the bacterial gene 16SrRNA (e.g., pyrosequensing) allowed significant progress in the knowledge of our microbial partners ${ }^{(5,6)}$. Even if most of the function of the microbial genes remains unknown until now, and if we are conditioned at birth with a 'personal' profile of gut microbes, several recent papers and reviews support the idea that 'dysbiosis' (inadequate change of gut microbiota composition and/or activity related to host disease) characterises obese or overweight individuals ${ }^{(7-11)}$.

The first studies reporting changes in gut microbiota composition in obese individuals have focused on changes in phyla proportion (decreased Bacteroides:Firmicutes ratio).
Recently, Arumugam et al. ${ }^{(12)}$ have identified, in individuals from different countries and continents, three robust clusters of gut microbial communities defined as 'enterotypes' by the authors. They found that these enterotypes were identified by the variation at the level of one of the three following genera: Bacteroides, Prevotella and Ruminococcus. Wu et al. ${ }^{(10)}$ have shown that enterotypes were strongly associated with long-term diet, namely protein and animal fat (associated with Bacteroides) $v$. carbohydrates (Prevotella). Experiments performed in a model of mice colonised with the human gut microbiota reveals that changes in the diet composition (from high carbohydrates to western diet) allows a rapid switch of the microbial community, which can be transferred to germ-free mice ${ }^{(13)}$. Interestingly, the transfer of this modified gut microbiota to germ-free mice also transfers the obese phenotype ${ }^{(13)}$. Those data suggest that the gut microbiota composition/activity associated with nutritional imbalance might contribute to obesity and related disorders ${ }^{(8,14-17)}$. If dysbiosis exists, is there a means to favourably change the microbial environment, and thereby to improve host health? This idea fits with the concept of prebiotic, originally described in the $90 \mathrm{~s}$, referring to dietary compounds that modulate the composition and activity of the gastrointestinal microbiota to improve health and wellbeing $^{(18-20)}$. The main purpose of the present paper is to report how nutrients with potential prebiotic properties are interacting with host metabolism in the context of obesity.

Abbreviation: LPS, lipopolysaccharides.

*Corresponding author: N. M. Delzenne, fax +32 276473 59; email nathalie.delzenne@uclouvain.be 
We will refer to both mechanistic approaches in animals and to the lower number of intervention studies performed with prebiotics in human subjects.

\section{Selection of nutrients with prebiotic properties: the bifidogenic effect as a starting point}

Wu et al. ${ }^{(10)}$ have shown that microbiome composition may change $24 \mathrm{~h}$ after initiating a high-fat/low-fibre or a highfibre/low-fat diet, but that enterotype identity remained stable during a $10 \mathrm{~d}$ nutritional intervention. They suggest that nutrients like dietary fibres, which are not digested by host enzymes, could modulate the gut microbiome in a relatively short period of time, independent of the effect of changes in transit time. Would it be possible to link the properties of dietary fibres, which specifically modulate the gut microbiota, with host functions related to obesity and overfeeding? Fermentable carbohydrates have initially been recognised as prebiotics, because they were preferentially fermented by specific types of bacteria, generally recognised as beneficial for host. Indeed, Bifidobacterium spp. represent an important and complex group of bacteria whose presence is often associated with interesting health effects ${ }^{(21-23)}$. In the context of obesity, several studies reported that a low number of Bifidobacterium spp. correlated with the development of obesity and/or diabetes ${ }^{(24-26)}$. We have previously demonstrated that diet-induced obesity (high-fat-low-carbohydrate diet) in mice markedly affects the gut microbial community, where the levels of Bifidobacterium spp. were significantly reduced, in accordance with the observation in human subjects $^{(27,28)}$. Several fermentable carbohydrates (glucans, galactans, fructans, etc.) are easily and widely fermented by Bifidobacteria. Several data have shown the bifidogenic effect of dietary fructans or arabinoxylans added in the diet of obese mice or rats ${ }^{(27,29-35)}$.

In fact, promoting Bifidobacteria is not the sole consequence of the prebiotic treatment. By pyrosequencing and microarray analysis of the caecal 16SrDNA of ob/ob mice treated or not with prebiotics, we were able to point out more than 100 taxa that were different upon prebiotic treatment, some bacteria being particularly increased and some of them decreased by more than $10-$ fold $^{(29)}$. This allowed for the identification of interesting bacteria which are promoted with a prebiotic approach in this particular context, such as Faecalibacterium prausnitzii, exhibiting interesting anti-inflammatory properties and potentially involved in diabetes-related inflammation, or Akkermansia muciniphila, which has been shown to be inversely correlate with weight gain ${ }^{(29,36,37)}$. Other studies have also shown that the prebiotic fibres decreased the Firmicutes:Bacteroidetes ratio in obese rats ${ }^{(34)}$. Concerning human data, we have recently confirmed, in an intervention study with prebiotics $v$. placebo in obese women that even if the increase in Bifidobacteria remains the major and common signature of the prebiotic approach, a complex modulation of the gut microbial ecology also occurs upon prebiotic treatment in obese individuals $^{(38)}$.
Effect of prebiotic on host metabolism in obesity: relation with the modulation of the microbial ecosystem

\section{Effect on body weight and adiposity}

In obese animals (ob/ob mice, diet-induced obesity, obese Zucker or JCR:LA-cp rats), the dietary supplementation with non-digestible/fermentable carbohydrates - such as inulintype fructans or arabinoxylans - is able to lessen adiposity $^{(16,30,33,34,39-41)}$. Prebiotic treatment changes the gene expression pattern in the white adipose tissue of obese mice (by acting on PPAR $\gamma$ and G-coupled receptors protein 43), leading to an increased lipolysis, a decreased adipogenesis and an increased metabolic response to hormones such as leptin, all those phenomenon contributing to a lower adiposity $^{(29,30,42)}$. In human subjects, treating obese individuals with fructan-type prebiotics has been tried in a limited number of intervention studies. Ingestion of inulin-type fructans prebiotic for 1 year has a significant benefit in the maintenance of BMI and fat mass in non-obese young adolescents $^{(43)}$. Three months of treatment with fructans also decreases body weight gain and fat mass in adult obese subjects ${ }^{(44)}$. The daily intake of yacon syrup, which contributed to an intake of $0 \cdot 14 \mathrm{~g}$ fructans $/ \mathrm{kg}$ per $\mathrm{d}$ over $120 \mathrm{~d}$, decreases body weight, waist circumference and BMI in obese premenopausal women ${ }^{(45)}$. Even if those data are significant, the weight loss remains modest (a few kg). None of those studies have reported the link between the changes in host adiposity and gut microbial composition.

\section{Effect on gut peptides and appetite regulation}

In obese animal fed inulin-type fructans, an increase in anorexigenic peptides (peptide YY and glucagon-like peptide 1 (7-36) amide) and a decrease in the orexigenic peptide ghrelin occurs, which contributes to the satietogenic effect of the peptide (for review see Cani \& Delzenne ${ }^{(15)}$ ). In addition, the supplementation with fructans in high-fat diet-fed mice modulates the neuronal activation within the arcuate nucleus, which can contribute to the control of food intake ${ }^{(35)}$. In human subjects, the satietogenic effect related to prebiotic interventions (assessed after 2 weeks to 3 months of treatment) is also being related to an increase in satietogenic and/or a decrease in orexigenic (ghrelin) peptides ${ }^{(44,46-48)}$.

The modulation of the gut endocrine function by prebiotics in obese mice involves an increase in the number of L endocrine cells in the intestine, an effect which is correlated to bacterial changes in the gut ${ }^{(29)}$. It is rather difficult to know by which mechanism the gut microbial environment influences L cells' differentiation. However, the production of SCFA (namely acetate, propionate) upon prebiotic fermentation could be part of the increase in secretion of gut peptides by the endocrine cells ${ }^{(49)}$.

\section{Effect on inflammation and gut barrier integrity}

The gut microbiota can be involved in the development of a low-grade inflammation, classically associated with the metabolic disorders related to obesity ${ }^{(28,50,51)}$. The serum level of 
lipopolysaccharides (LPS), the main component of the Gramnegative bacteria, is approximately doubled in obese, diabetic or high-fat diet-fed individuals, a phenomenon that contributes to proinflammatory processes. The increase in LPS may occur by processes involving an increase in chylomicron formation (upon high-fat diet feeding), a decrease in gut barrier integrity and/or a decrease in alkaline phosphatase activity, which is the enzyme responsible for the cleavage of LPS in the intestine (for review see Cani \& Delzenne ${ }^{(15)}$ and Cani et $a l{ }^{(52)}$ ). Several prebiotics (glucans, fructans) are able to counteract the increase in LPS level in animal models of obesity $^{(27,31,32,53)}$. The decrease in LPS absorption occurs in prebiotic-treated animals through an improvement of the expression and activity of proteins involved in gut barrier function, including glucagon-like peptide 2, which is co-secreted with glucagon-like peptide 1 by endocrine L cells. In addition, a drop in endocannabinoid system activation in the intestinal cells also participates in the gut barrier function by prebiotics in obese animals ${ }^{(27,32,39,46,54-58)}$. Further mechanistic studies are needed in order to better understand how prebiotic nutrients may interact with the host immune response in the context of obesity and related disorders. Moreover, the relevance of those effects remains to be studied in human subjects.

\section{Effect on glucose and lipid metabolism}

In the majority of studies, the administration of prebiotics lead to an improvement of fasting and/or post-oral glucose load glycaemia (for review see Roberfroid et al. ${ }^{(19)}$ ). The mechanisms could involve the secretion of gut peptides with incretin function, such as glucagon-like peptide 1, which participates in the improvement of hepatic insulin resistance ${ }^{(57)}$. Several studies in human subjects also show an improvement in postprandial glycaemia, or, in some studies, in triglyceridaemia, upon prebiotic treatment, but those data are really not numerous enough to draw any conclusion on a potential benefit for diabetic or dyslipidaemic patients (for review see Delzenne et $\left.a l .{ }^{(41)}\right)$. In most experimental studies, prebiotics are able to decrease the hepatic accumulation of TAG and/ or cholesterol in the liver tissue, defined as steatosis. This effect could be particularly interesting, as the occurrence of non-alcoholic fatty liver disease is present in $25-75 \%$ of the obese individuals. There again, mechanistic studies in animals reveal changes in hepatic host gene expression upon prebiotic treatment that could implicate, depending on the experimental conditions, a decrease in sterol-response-element-binding protein-dependent cholesterogenesis and/or lipogenesis, and/or of changes in PPAR $\alpha$-driven fatty acid oxidation (for review see Delzenne et $a l^{(41)}$, Delzenne \& $\mathrm{Cani}^{(59)}$ and Pachikian et al. $\left.{ }^{(60)}\right)$. Once again, research is needed to discover which microbial-derived metabolite could interfere with those metabolic processes. Only two intervention studies with prebiotics (fructans) have been reported in patients exhibiting hepatic diseases, suggesting an improvement of markers such as LPS or aminotransferases, without referring to the modulation of the gut microbiota ${ }^{(61,62)}$.

\section{Conclusion and perspectives}

Highly fermentable carbohydrates, such as prebiotics, are able to counteract several metabolic alterations linked to obesity, including hyperglycaemia, inflammation and hepatic steatosis, at least in animal models (Fig. 1). The mechanistic studies suggest that the changes in the gut microbiota occurring upon prebiotic treatment, which appear much wider than the single increase in Bifidobacteria initially described, can be related to an improvement of gut bacterial functions implicated in the regulation of host energy homoeostasis. The promotion of gut hormones' release, changes in the gut barrier integrity and/or the release of bacterial-derived metabolites
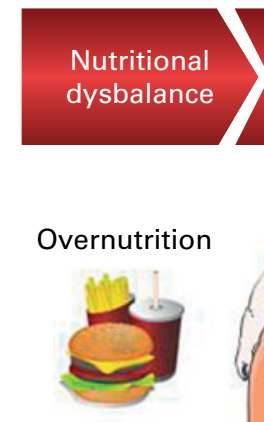

High lipids High carbohydrates $n$-3 PUFA deficiency

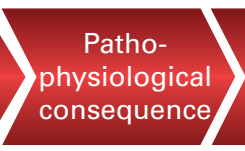

Metabolic disorders
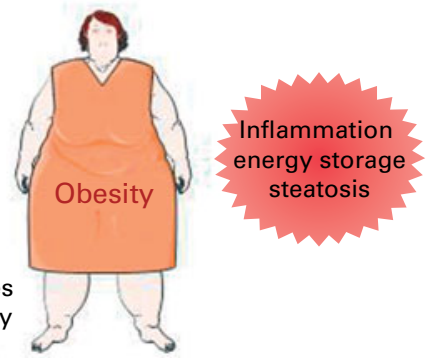
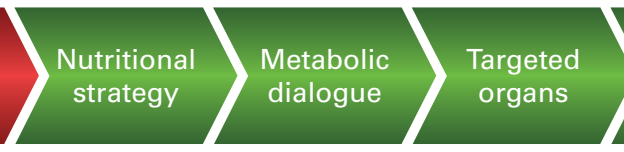

Molecular targets
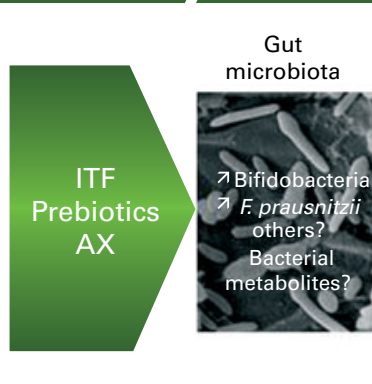

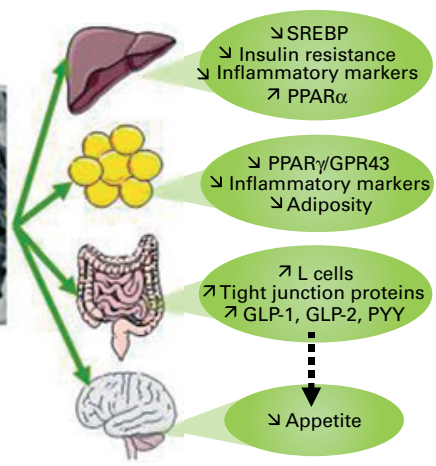

Fig. 1. Effect of dietary carbohydrates with prebiotic properties on host pathophysiology related to obesity. In view of the experimental data obtained in intervention studies in animals, it has been shown that dietary carbohydrates with prebiotic properties change the gut microbiota composition by favouring bacteria involved in the control of gut barrier function and host immunity. In the gut, prebiotics help reinforcing the gut barrier and promote gut hormones that control appetite, glucose homoeostasis and systemic inflammation. The prebiotic approach also counteracts hepatic steatosis, hepatic insulin resistance and adiposity by modifying gene expression at the tissue level. F. prausnitzii, Faecalibacterium prausnitzii; SREBP, sterol-regulatory-element-binding protein; GPR43, G-coupled receptors protein 43; GLP, glucagon-like peptide; PYY, peptide YY. ITF, inulin-type fructans; AX, arabinoxylans. 
could all participate in the improvement of host health in the particular context of overfeeding and obesity. Appropriate human intervention studies with 'colonic' nutrients (dietary fibres, prebiotics and others) able to selectively promote beneficial bacteria, or with food containing colonic nutrients, are essential to confirm the relevance of those nutrients in the nutritional management of overweight and obesity.

\section{Acknowledgements}

P. D. C. is a research associate from the FRS-FNRS (Fonds de la Recherche Scientifique, Belgique). N. M. D. and P. D. C. are recipients of subsides from the Fonds National de la Recherche Scientifique (FNRS/FRSM) and from the 'fonds spéciaux de recherche', Université catholique de Louvain (UCL). The authors declare no conflict of interest related to the content of the present paper. All authors contributed equally to all aspects of the article.

\section{References}

1. Hotamisligil GS (2006) Inflammation and metabolic disorders. Nature 444, 860-867.

2. Gil A, Ortega RM \& Maldonado J (2011) Wholegrain cereals and bread: a duet of the Mediterranean diet for the prevention of chronic diseases. Public Health Nutr 14, 2316-2322.

3. Qin J, Li R, Raes J, et al. (2010) A human gut microbial gene catalogue established by metagenomic sequencing. Nature 464, 59-65.

4. Diamant M, Blaak EE \& de Vos WM (2011) Do nutrient-gutmicrobiota interactions play a role in human obesity, insulin resistance and type 2 diabetes? Obes Rev 12, 272-281.

5. Hsiao WW \& Fraser-Liggett CM (2009) Human microbiome project - paving the way to a better understanding of ourselves and our microbes. Drug Discov Today 14, 331-333.

6. Turnbaugh PJ, Ley RE, Hamady M, et al. (2007) The human microbiome project. Nature 449, 804-810.

7. Ley RE, Backhed F, Turnbaugh P, et al. (2005) Obesity alters gut microbial ecology. Proc Natl Acad Sci U S A 102, 11070-11075.

8. Ley RE (2010) Obesity and the human microbiome. Curr Opin Gastroenterol 26, 5-11.

9. Ley RE, Turnbaugh PJ, Klein S, et al. (2006) Microbial ecology: human gut microbes associated with obesity. Nature 444, 1022-1023.

10. Wu GD, Chen J, Hoffmann C, et al. (2011) Linking long-term dietary patterns with gut microbial enterotypes. Science $\mathbf{3 3 4}$ 105-108.

11. Munukka E, Wiklund P, Pekkala S, et al. (2012) Women with and without metabolic disorder differ in their gut microbiota composition. Obesity (Silver Spring) 20, 1082-1087.

12. Arumugam M, Raes J, Pelletier E, et al. (2011) Enterotypes of the human gut microbiome. Nature 473, 174-180.

13. Turnbaugh PJ, Ridaura VK, Faith JJ, et al. (2009) The effect of diet on the human gut microbiome: a metagenomic analysis in humanized gnotobiotic mice. Sci Transl Med 1, Gra14.

14. Caesar R, Fak F \& Backhed F (2010) Effects of gut microbiota on obesity and atherosclerosis via modulation of inflammation and lipid metabolism. J Intern Med 268, 320-328.

15. Cani PD \& Delzenne NM (2011) The gut microbiome as therapeutic target. Pharmacol Ther 130, 202-212.

16. Delzenne NM \& Cani PD (2010) Nutritional modulation of gut microbiota in the context of obesity and insulin resistance: potential interest of prebiotics. Int Dairy $J \mathbf{2 0}$, 277-280.

17. Kau AL, Ahern PP, Griffin NW, et al. (2011) Human nutrition, the gut microbiome and the immune system. Nature $\mathbf{4 7 4}$, $327-336$.

18. Gibson GR \& Roberfroid MB (1995) Dietary modulation of the human colonic microbiota: introducing the concept of prebiotics. J Nutr 125, 1401-1412.

19. Roberfroid M, Gibson GR, Hoyles L, et al. (2010) Prebiotic effects: metabolic and health benefits. Br J Nutr 104, Suppl. 2, S1-S63.

20. Brownawell AM, Caers W, Gibson GR, et al. (2012) Prebiotics and the health benefits of fiber: current regulatory status, future research, and goals. J Nutr 142, 962-974.

21. Boesten RJ \& de Vos WM (2008) Interactomics in the human intestine: Lactobacilli and Bifidobacteria make a difference. J Clin Gastroenterol 42, S163-S167.

22. Boesten RJ, Schuren FH \& de Vos WM (2009) A Bifidobacterium mixed-species microarray for high resolution discrimination between intestinal bifidobacteria. J Microbiol Methods 76, 269-277.

23. Turroni F, Marchesi JR, Foroni E, et al. (2009) Microbiomic analysis of the bifidobacterial population in the human distal gut. ISME J 3, 745-751.

24. Kalliomaki M, Collado MC, Salminen S, et al. (2008) Early differences in fecal microbiota composition in children may predict overweight. Am J Clin Nutr 87, 534-538.

25. Collado MC, Isolauri E, Laitinen K, et al. (2008) Distinct composition of gut microbiota during pregnancy in overweight and normal-weight women. Am J Clin Nutr 88, 894-899.

26. Wu X, Ma C, Han L, et al. (2010) Molecular characterisation of the faecal microbiota in patients with type II diabetes. Curr Microbiol 61, 69-78.

27. Cani PD, Neyrinck AM, Fava F, et al. (2007) Selective increases of bifidobacteria in gut microflora improve highfat-diet-induced diabetes in mice through a mechanism associated with endotoxaemia. Diabetologia 50, 2374-2383.

28. Cani PD, Amar J, Iglesias MA, et al. (2007) Metabolic endotoxemia initiates obesity and insulin resistance. Diabetes 56, 1761-1772.

29. Everard A, Lazarevic V, Derrien M, et al. (2011) Responses of gut microbiota and glucose and lipid metabolism to prebiotics in genetic obese and diet-induced leptin-resistant mice. Diabetes 60, 2775-2786.

30. Neyrinck AM, Possemiers S, Druart C, et al. (2011) Prebiotic effects of wheat arabinoxylan related to the increase in bifidobacteria, Roseburia and Bacteroides/Prevotella in dietinduced obese mice. PLoS One 6, e20944.

31. Neyrinck AM, Van Hée VF, Piront N, et al. (2012) Wheatderived arabinoxylan oligosaccharides with prebiotic effect increase satietogenic gut peptides and reduce metabolic endotoxemia in diet-induced obese mice. Nutr Diabetes 2, e28.

32. Cani PD, Possemiers S, Van de WT, et al. (2009) Changes in gut microbiota control inflammation in obese mice through a mechanism involving GLP-2-driven improvement of gut permeability. Gut 58, 1091-1103.

33. Parnell JA \& Reimer A (2012) Prebiotic fiber modulation of the gut microbiota improves risk factors for obesity and the metabolic syndrome. Gut Microbes 3, 29-34.

34. Parnell JA \& Reimer RA (2012) Prebiotic fibres dosedependently increase satiety hormones and alter Bacteroidetes and Firmicutes in lean and obese JCR:LA-cp rats. $\mathrm{Br} \mathrm{J}$ Nutr 107, 601-613.

35. Anastasovska J, Arora T, Sanchez Canon GJ, et al. (2012) Fermentable carbohydrate alters hypothalamic neuronal activity 
and protects against the obesogenic environment. Obesity (Silver Spring) 20, 1016-1023.

36. Santacruz A, Collado MC, Garcia-Valdes L, et al. (2010) Gut microbiota composition is associated with body weight, weight gain and biochemical parameters in pregnant women. Br J Nutr 104, 83-92.

37. Furet JP, Kong LC, Tap J, et al. (2010) Differential adaptation of human gut microbiota to bariatric surgery-induced weight loss: links with metabolic and low-grade inflammation markers. Diabetes 59, 3049-3057.

38. Dewulf EM, Cani PD, Claus SP, et al. (2012) Inulin-type fructans with prebiotic properties lessen endotoxemia and modulate host metabolism by changing gut microbiota composition in obese women. Obes Facts Eur J Obes 5, 200.

39. Cani PD \& Delzenne NM (2009) The role of the gut microbiota in energy metabolism and metabolic disease. Curr Pharm Des 15, 1546-1558.

40. Neyrinck AM \& Delzenne NM (2010) Potential interest of gut microbial changes induced by non-digestible carbohydrates of wheat in the management of obesity and related disorders. Curr Opin Clin Nutr Metab Care 13, 722-728.

41. Delzenne NM, Neyrinck AM, Backhed F, et al. (2011) Targeting gut microbiota in obesity: effects of prebiotics and probiotics. Nat Rev Endocrinol 7, 639-646.

42. Dewulf EM, Cani PD, Neyrinck AM, et al. (2011) Inulin-type fructans with prebiotic properties counteract GPR43 overexpression and PPARgamma-related adipogenesis in the white adipose tissue of high-fat diet-fed mice. J Nutr Biochem 22, $712-722$.

43. Abrams SA, Griffin IJ, Hawthorne KM, et al. (2007) Effect of prebiotic supplementation and calcium intake on body mass index. J Pediatr 151, 293-298.

44. Parnell JA \& Reimer RA (2009) Weight loss during oligofructose supplementation is associated with decreased ghrelin and increased peptide YY in overweight and obese adults. Am J Clin Nutr 89, 1751-1759.

45. Genta S, Cabrera W, Habib N, et al. (2009) Yacon syrup: beneficial effects on obesity and insulin resistance in humans. Clin Nutr 28, 182-187.

46. Cani PD, Lecourt E, Dewulf EM, et al. (2009) Gut microbiota fermentation of prebiotics increases satietogenic and incretin gut peptide production with consequences for appetite sensation and glucose response after a meal. Am J Clin Nutr $\mathbf{9 0}$, $1236-1243$.

47. Cani PD, Joly E, Horsmans Y, et al. (2006) Oligofructose promotes satiety in healthy human: a pilot study. Eur J Clin Nutr 60, 567-572.

48. Verhoef SP, Meyer D \& Westerterp KR (2011) Effects of oligofructose on appetite profile, glucagon-like peptide 1 and peptide YY3-36 concentrations and energy intake. $\mathrm{Br} \mathrm{J}$ Nutr 106, 1757-1762.
49. Tolhurst G, Heffron H, Lam YS, et al. (2012) Short-chain fatty acids stimulate glucagon-like peptide-1 secretion via the G-protein-coupled receptor FFAR2. Diabetes 61, 364-371.

50. de Lartigue G, de La Serre CB \& Raybould HE (2011) Vagal afferent neurons in high fat diet-induced obesity; intestinal microflora, gut inflammation and cholecystokinin. Physiol Behav 105, 100-105.

51. Reigstad CS, Lunden GO, Felin J, et al. (2009) Regulation of serum amyloid A3 (SAA3) in mouse colonic epithelium and adipose tissue by the intestinal microbiota. PLoS One $\mathbf{4}$, e5842.

52. Cani PD, Osto M, Geurts L, et al. (2012) Involvement of gut microbiota in the development of low-grade inflammation and type 2 diabetes associated with obesity. Gut Microbes 1; 3(4). http://dx.doi.org/10.4161/gmic.19625.

53. Serino M, Luche E, Gres S, et al. (2012) Metabolic adaptation to a high-fat diet is associated with a change in the gut microbiota. Gut 61, 543-553.

54. Cani PD \& Delzenne NM (2009) Interplay between obesity and associated metabolic disorders: new insights into the gut microbiota. Curr Opin Pharmacol 9, 737-743.

55. Delzenne NM, Cani PD \& Neyrinck AM (2007) Modulation of glucagon-like peptide 1 and energy metabolism by inulin and oligofructose: experimental data. J Nutr 137, 2547S-2551S.

56. Cani PD \& Delzenne NM (2007) Gut microflora as a target for energy and metabolic homeostasis. Curr Opin Clin Nutr Metab Care 10, 729-734.

57. Cani PD, Knauf C, Iglesias MA, et al. (2006) Improvement of glucose tolerance and hepatic insulin sensitivity by oligofructose requires a functional glucagon-like peptide 1 receptor. Diabetes 55, 1484-1490.

58. Muccioli GG, Naslain D, Backhed F, et al. (2010) The endocannabinoid system links gut microbiota to adipogenesis. Mol Syst Biol 6, 392.

59. Delzenne NM \& Cani PD (2011) Interaction between obesity and the gut microbiota: relevance in nutrition. Annu Rev Nutr 31, 15-31.

60. Pachikian BD, Essaghir A, Demoulin JB, et al. (2011) Prebiotic approach improves hepatic steatosis associated with $n-3$ polyunsaturated fatty acid depletion in mice. Ann Nutr Metab 58, S3, 76.

61. Daubioul CA, Horsmans Y, Lambert P, et al. (2005) Effects of oligofructose on glucose and lipid metabolism in patients with nonalcoholic steatohepatitis: results of a pilot study. Eur J Clin Nutr 59, 723-726.

62. Malaguarnera M, Vacante M, Antic T, et al. (2012) Bifidobacterium longum with fructo-oligosaccharides in patients with non alcoholic steatohepatitis. Dig Dis Sci 57, 545-553. 